\section{СТОЙКАЯ МАРКИРОВКА}

\section{С ИСПОЛЬЗОВАНИЕМ ЛАЗЕРОВ}

\author{
А.Щейбер, Datalogic Group
}

\begin{abstract}
В обзоре рассмотрены системы лазерной маркировки. Даны примеры их использования в промышленном производстве и предложены критерии, позволяющие принять решение при выборе этих систем и реализаций конкретных методов, учитывая их преимущества по сравнению с другими методами маркировки. Также представлены технические характеристики маркировочных устройств, предъявляемые к ним требования и технологии с их использованием.
\end{abstract}

азерная маркировка - это надежная и универсальная технология. Ее можно использовать в самых различных областях применения - при нанесении срока годно сти на бутылки с водой, фирменной символики на мобильные телефоны, при маркировке приборной панели автомобилей с подсветкой и при создании бирок для крупного рогатого скота. Лазерная маркировка просто необходима как для товаров широкого потребления, так и для промышленной продукции, когда речь идет о нанесении номеров партии или серии, срока годности, официальных логотипов и прочей информации.

Благодаря уникальным эксплуатационным параметрам лазерные устройства обеспечивают чрезвычайно надежную, универсальную и автоматизированную маркировку, отличающуюся стойкостью и долговременностью. Во многих отраслях, таких как автомобильная, электрон ная, пищевая и медицинская промышлен ность, а также индустрия моды используются технологии лазерной маркировки для надеж ной идентификации, защищающей от подде лок, нанесения фирменной символики, без опасности продукта и его отслеживания. Благодаря использованию устройств лазерной маркировки производители повышают эффек тивность и точность производства, а также обе спечивают высочайшую точность маркировки изделий. В результате маркировка полностью соответствует строжайшим гигиеническим стандартам промышленных изделий и более благоприятна для окружающей среды вслед-

\section{PERMANENT LABELLING}

\section{WITH LASERS}

\author{
A. Schaber, Datalogic Group
}

This whitepaper will discuss the advantages of Laser Marking Systems, examples of use in the manufacturing industry and the decision criteria for choosing a laser marking product in comparison to other labelling methods. Laser marking device features, requirements and the technology behind different marking methods will also be discussed.

aser marking is a permanent and flexible technology that can be used in a variety of applications such as expiration dates on water bottles, mobile phone branding, backlighted car dashboards and cattle tags. Whether batch, serialized number, best before date, official logos, or other data, consumer products and industrial items cannot exist without laser marking.

With its unique performance, laser marking guarantees extremely reliable, flexible and automated marking that is permanent and durable. Numerous industries such as automotive, electronic, fashion, food and medical rely on laser marking for reliable and counterfeit proof identification, labelling, branding, product security and backtracking purposes. Through the use of laser marking devices, manufacturers increase production efficiency, achieve precision quality and highly accurate labelling of work pieces. The resulting mark fully complies with the highest hygienic standards for manufactured goods and a better ecological footprint due to reduced waste and use of chemicals.

Laser marking delivers an economical and effective solution for permanent labelling of every type of surface. It withstands the most rugged conditions, guarantees backtracking over the

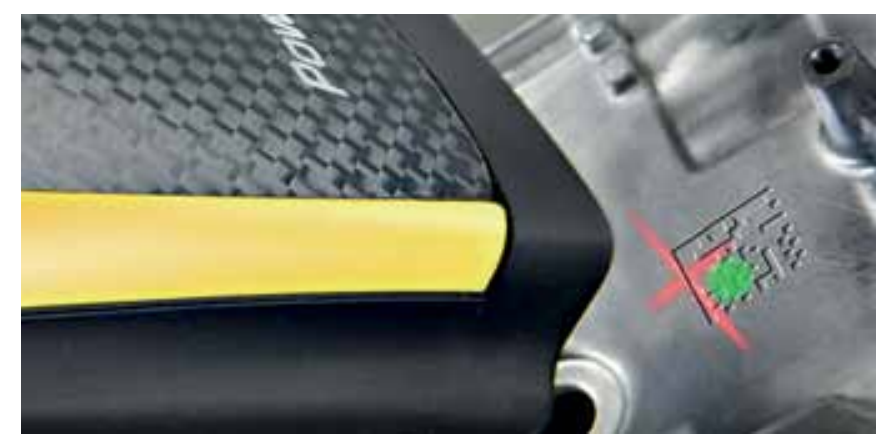




\section{TECHNOLOGY EQUIPMENT AND TECHNOLOGIES}

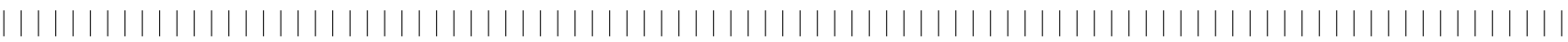

ствие сокращения количества отходов и отсутствия химических веществ.

Лазерная маркировка представляет собой экономичное и эффективное решение для нанесения стойких информационных знаков на любую поверхность. Она устойчива к самым жестким условиям, гарантирует отслеживание продукции на протяжении всего срока службы, позволяет учитывать пожелания entire product life cycle, allows individualization and assists in quality assurance and protection against counterfeiting. Laser marking offers many advantages in comparison to other methods, but choosing to use it depends on many factors, including whether its advantages can be realized completely. This whitepaper provides information that can be used as a guideline for adopting a laser marking solution.

Таблица 1. Сравнение различных методов маркировки и этикетирования по параметрам

Table 1. Marking and Labelling Methods Comparison

\begin{tabular}{|c|c|c|c|c|c|c|}
\hline \multirow[b]{2}{*}{ Параметр } & \multirow[b]{2}{*}{$\begin{array}{l}\text { Лазерная } \\
\text { маркировка } \\
\text { Laser } \\
\text { Marking }\end{array}$} & \multirow[b]{2}{*}{$\begin{array}{l}\text { Ударно- } \\
\text { точечная } \\
\text { маркировка } \\
\text { DotPeen }\end{array}$} & \multirow[b]{2}{*}{$\begin{array}{l}\text { Игольчатая } \\
\text { маркировка } \\
\text { Stylus } \\
\text { Marking }\end{array}$} & \multicolumn{3}{|c|}{ Печать } \\
\hline & & & & $\begin{array}{l}\text { Непрерывная } \\
\text { струйная } \\
\text { печать } \\
\text { Continuous } \\
\text { Ink-Jet } \\
\text { Printing }\end{array}$ & $\begin{array}{l}\text { Печать } \\
\text { этикеток } \\
\text { Label } \\
\text { Printing }\end{array}$ & $\begin{array}{c}\text { Термо- } \\
\text { трансферная } \\
\text { и тампо- } \\
\text { печать } \\
\text { Thermo- } \\
\text { Transfer } \\
\text { Pad printing }\end{array}$ \\
\hline $\begin{array}{l}\text { Стабильность } \\
\text { Contrast }\end{array}$ & $\uparrow$ & $\uparrow$ & $\uparrow$ & $\leftrightarrow$ & $\downarrow$ & $\downarrow$ \\
\hline $\begin{array}{l}\text { Контрастность } \\
\text { Contrast }\end{array}$ & $\leftrightarrow$ & $\downarrow$ & $\downarrow$ & $\uparrow \uparrow$ & $\uparrow \uparrow$ & 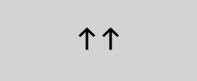 \\
\hline $\begin{array}{l}\text { Отсутствие расходных } \\
\text { материалов } \\
\text { Consumable free }\end{array}$ & $\uparrow \uparrow$ & $\uparrow$ & $\uparrow$ & $\downarrow \downarrow$ & $\downarrow \downarrow$ & $\downarrow \downarrow$ \\
\hline $\begin{array}{l}\text { Отсутствие техниче- } \\
\text { ского обслуживания } \\
\text { Maintenance free }\end{array}$ & 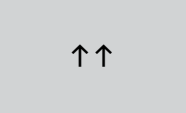 & $\uparrow$ & $\uparrow$ & $\downarrow \downarrow$ & $\uparrow$ & $\leftrightarrow$ \\
\hline $\begin{array}{l}\text { Требования } \\
\text { безопасности } \\
\text { Safety Requirements }\end{array}$ & $\uparrow$ & $\downarrow$ & $\downarrow$ & $\downarrow$ & $\downarrow$ & $\downarrow$ \\
\hline $\begin{array}{l}\text { Экологическая безопас- } \\
\text { ность } \\
\text { Environmental friendly }\end{array}$ & 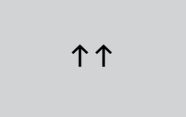 & 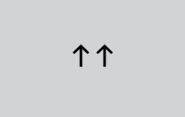 & 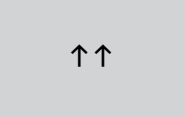 & $\downarrow$ & $\leftrightarrow$ & $\downarrow$ \\
\hline $\begin{array}{l}\text { Время обработки } \\
\text { Process Time }\end{array}$ & $\uparrow$ & $\downarrow$ & $\downarrow$ & $\uparrow$ & $\leftrightarrow$ & $\uparrow$ \\
\hline $\begin{array}{l}\text { Затраты на единицу про- } \\
\text { дукции } \\
\text { Unit Cost }\end{array}$ & $\downarrow$ & $\downarrow$ & $\downarrow$ & $\leftrightarrow$ & 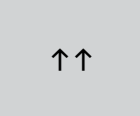 & $\leftrightarrow$ \\
\hline $\begin{array}{l}\text { Начальные затраты } \\
\text { Initial Cost }\end{array}$ & $\uparrow$ & $\downarrow$ & $\downarrow$ & $\leftrightarrow$ & $\downarrow$ & $\leftrightarrow$ \\
\hline
\end{tabular}

Условные обозначения:

\footnotetext{
$\uparrow \quad$ Преимущество метода $\uparrow \uparrow$ Усиленное преимущество

$\downarrow$ Недостатки метода $\quad \downarrow \downarrow$ Усиленные недостатки

$\leftrightarrow$ Не имеет значения
} 
заказчика и обеспечивает качество продукции и ее защиту от подделок. Лазерная марки ровка имеет множество преимуществ по срав нению с другими методами, однако принятие решения о ее использовании зависит от многих факторов, в том числе, следует учитывать, могут ли все преимущества такой маркировки полностью быть реализованы в той или иной ситуации. В настоящем обзоре представлена информация, которая может служить руководством при выборе технологии лазерной маркировки.

\section{ТЕХНОЛОГИИ, ЛЕЖАЩИЕ В ОСНОВЕ СИСТЕМ МАРКИРОВКИ (ТАБЛ. 1 И 2) Лазерная маркировка}

Лазерная маркировка позволяет с большой скоростью наносить на поверхность изображения с высочайшей точностью. Эта потрясающая технология использует электромагнитное излу-

\section{THE TECHNOLOGY BEHIND MARKING SYSTEMS \\ Laser Marking}

Laser marking delivers high speed permanent images with pinpoint accuracy. The power behind this incredible technology is light used as electromagnetic energy. Light visible by the human eye is in the wave spectrum 380-700 nanometer (nm). Laser marking systems generally use the intense light energy of visible or invisible wavelengths, such as infrared (> $800 \mathrm{~nm})$ und UV $(<380 \mathrm{~nm})$, for permanent modification of target object surfaces.

A collimated laser beam is precisely and accurately positioned and focused by moving mirrors and special optical devices on the surface of a treated object. Depending on the characteristics of the laser source, the surface is struck and modified by extremely intense optical power. Since there is no use of additional layers, labels or inks, this process is called Direct Part Marking (DPM). Information

Таблица 2. Обработка поверхности с помощью лазерной технологии в зависимости от материала

Table 2. Laser Surface Treatments vs Materials

\begin{tabular}{|c|c|c|c|c|c|c|c|}
\hline & $\begin{array}{l}\text { Железо/ } \\
\text { Сталь } \\
\text { Iron/ } \\
\text { Steel }\end{array}$ & $\begin{array}{l}\text { Титан } \\
\text { Titan }\end{array}$ & $\begin{array}{l}\text { Метал } \\
\text { Metal }\end{array}$ & $\begin{array}{l}\text { Термо- } \\
\text { пласты } \\
\text { Thermo- } \\
\text { plastics }\end{array}$ & $\begin{array}{c}\text { Бумага, дерево, } \\
\text { органические } \\
\text { материалы } \\
\text { Paper, Wood, } \\
\text { Organic Material }\end{array}$ & $\begin{array}{l}\text { Стекло и про- } \\
\text { зрачные } \\
\text { материалы } \\
\text { Glass \& } \\
\text { Transparent } \\
\text { Materials }\end{array}$ & $\begin{array}{c}\text { Любые поверхно- } \\
\text { сти в зависимо- } \\
\text { сти от покрытия } \\
\text { All surfaces, } \\
\text { depending on } \\
\text { coating }\end{array}$ \\
\hline $\begin{array}{l}\text { Термообработка } \\
\text { Heat Treatment }\end{array}$ & $x$ & $x$ & - & - & - & - & - \\
\hline $\begin{array}{l}\text { Лазерная гравировка } \\
\text { Laser Engraving }\end{array}$ & $x$ & $x$ & $x$ & $x$ & $x$ & $\times$ & $\times$ \\
\hline $\begin{array}{l}\text { Лазерное травление } \\
\text { Laser Etching }\end{array}$ & - & - & $x$ & - & - & - & - \\
\hline $\begin{array}{l}\text { Импульсное лазерное } \\
\text { напыление } \\
\text { Pulsed Laser Deposition }\end{array}$ & - & - & - & - & - & - & $x$ \\
\hline $\begin{array}{l}\text { Вспенивание } \\
\text { Foaming }\end{array}$ & - & - & - & $x$ & - & - & - \\
\hline $\begin{array}{l}\text { Изменение цвета, нитри- } \\
\text { фикация, обесцвечивание } \\
\text { Change in color, } \\
\text { Nitrification, Bleaching }\end{array}$ & - & - & - & $x$ & - & - & - \\
\hline $\begin{array}{l}\text { Карбонизация } \\
\text { Carbonation }\end{array}$ & - & - & - & $x$ & $\times$ & - & - \\
\hline $\begin{array}{l}\text { Лазерная гравировка вну- } \\
\text { тренних поверхностей } \\
\text { Inside Laser Engraving }\end{array}$ & - & - & - & - & - & $x$ & - \\
\hline
\end{tabular}

Условные обозначения:

Не подходит для перечисленных материалов

× Подходит для перечисленных материалов 
чение светового диапазона. Свет, видимый человеческим глазом, находится в волновом диапазоне 380-700 (нм). Системы лазерной маркировки обычно используют интенсивную световую энергию видимой или невидимой части волнового спектра, например, инфракрасную (>800 нм) и ультрафиолетовую (UV) (< 380 нм), для постоянной модификации поверхности обрабатываемого объекта.

\section{Прямая маркировка деталей}

С помощью подвижных зеркал и специальных оптических устройств коллимированный лазерный пучок направляется и фокусируется с высочайшей точностью на поверхности обрабатываемого объекта. В зависимости от характеристик источника лазерного излучения поверхность объекта изменяется под воздействием лучевой энергии высокой интенсивности. Так как при этом не используются дополнительные комплектующие (слои, метки или чернила), то это процесс получил название "прямая маркировка деталей (Direct Part Marking (DPM). Информация, изображения, текст, одномерные или двумерные коды наносятся непосредственно на поверхность изделия, подлежащего маркировке.

Имеются также другие специальные процессы маркировки с использованием различных лазерных технологий и излучения разной интенсивности: травление, гравировка, изменение цвета поверхности, выжигание и карбонизация, снятие поверхностного слоя и т.д.
Таблица 3. Лазерная маркировка различных материалов

Table 3. Laser Marking for Various Materials

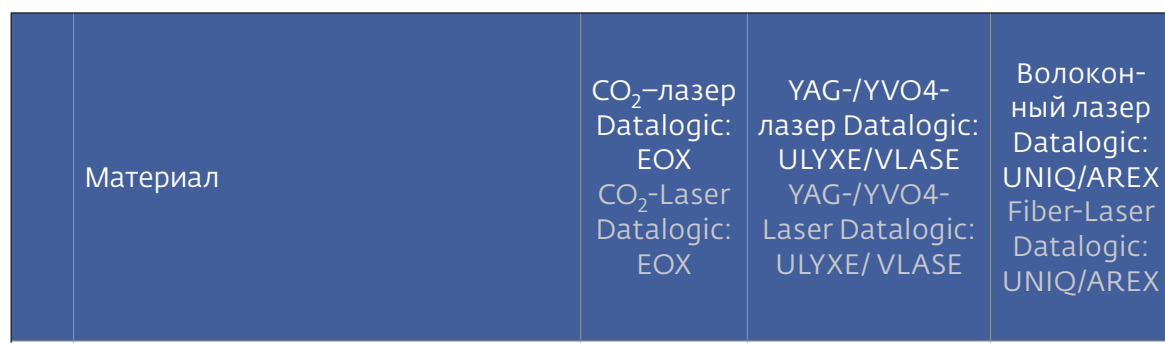

Полиэтилен (РE)

Polyethylene (PE)

\begin{tabular}{l|l|l}
++ & ++ & ++
\end{tabular}

Поликарбонат (РС)

Polycarbonate $(P C)$

$++$

$++$

Полипропилен (РP)

Polypropylene (PP)

Полиацеталь (РОМ)

Polyacetal (POM)

\begin{tabular}{l|l|l}
++ & +++ & +++
\end{tabular}

Полибутилентерефталат (РВТ)

Polybutylene terephthalate (PBT)

Полиэтилентерефталат (РЕТ)

Polyethylene terephthalate (PET)

$\overline{0}$

$\underset{v}{0}$

Акрилнитрилбутадиенстирол (ABS)

Acrylonitrile-butadiene-styrene (ABS)

Эпоксид

Epoxide

$+++$

$+++$

$+++$

Фенол

Phenol

Поливинилхлорид

Polyvinyl chloride

$+++$

$+++$

$+++$

Полиамид(РА)

Polyamide (PA)

Силикон

Silicone 


\section{Ударно-точечная маркировка}

Ударно-точечная маркировка осуществляется механическим движением прочной или твердосплавной иглы или бойка по поверхности. Каждый удар иглы образует точку на поверхности. С помощью точек создается буквенно-цифровой текст, штрих-коды или другие изображения. Данный метод подходит только для изделий с повышенной прочностью, например, изготовленных из стали или алюминия, а также для литых заготовок.

\section{Маркировка с помощью игольчатых устройств}

Маркировка с помощью игольчатых устройств схожа с ударно-точечной в том отношении, что она использует прочную или твердосплавную иглу для маркировки поверхности детали. Игольчатые устройства движутся по поверхности, оставляя непрерывную линию вместо точек. Буквенно-цифровые тексты, штрих-коды или другие изображения создаются путем контроля процесса соприкосновения иглы с поверхностью. Этот вид маркировки используется для изделий из металла, пластмассы и некоторых других материалов.

\section{Печатающие системы: струйные, этикеточные, термотрансферные}

Печатающие системы не меняют поверхность маркируемых изделий. Эти методы предполагают размещение необходимой информации на поверхности изделий посредством таких материалов, как чернила, этикетки или термотрансферная пленка.
Таблица 3. Продолжение

Table 3. Continuation

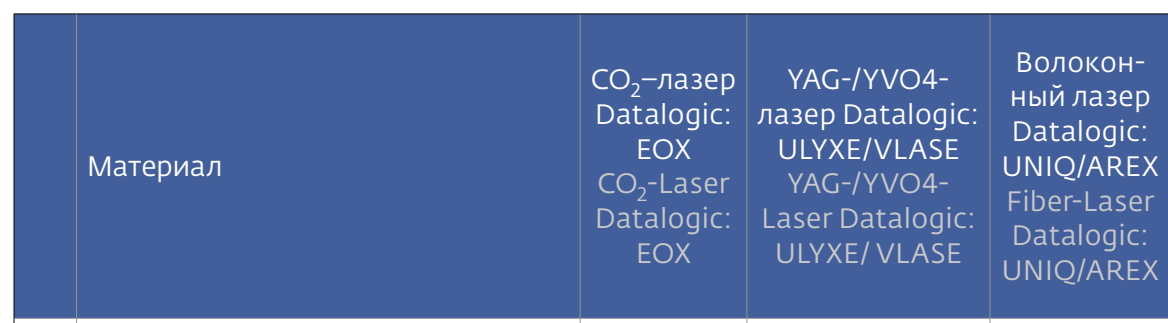

\begin{tabular}{|c|c|c|c|c|}
\hline \multirow{6}{*}{ 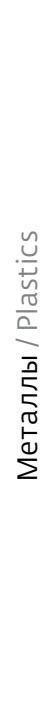 } & $\begin{array}{l}\text { Железо/Сталь } \\
\text { Iron/Steel }\end{array}$ & - & +++ & +++ \\
\hline & $\begin{array}{l}\text { Алюминий } \\
\text { Aluminium }\end{array}$ & - & +++ & +++ \\
\hline & $\begin{array}{l}\text { Никель } \\
\text { Nickel }\end{array}$ & - & +++ & +++ \\
\hline & $\begin{array}{l}\text { Нержавеющая сталь } \\
\text { Stainless steel }\end{array}$ & - & ++ & +++ \\
\hline & $\begin{array}{l}\text { Медь } \\
\text { сорper }\end{array}$ & - & ++ & + \\
\hline & $\begin{array}{l}\text { Золото } \\
\text { Gold }\end{array}$ & - & ++ & + \\
\hline \multirow{6}{*}{ 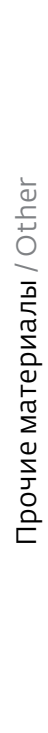 } & $\begin{array}{l}\text { Керамика } \\
\text { Ceramic }\end{array}$ & ++ & + & ++ \\
\hline & $\begin{array}{l}\text { Oрганические материалыс } \\
\text { Organic }\end{array}$ & +++ & - & - \\
\hline & $\begin{array}{l}\text { Дерево } \\
\text { Wood }\end{array}$ & +++ & - & - \\
\hline & $\begin{array}{l}\text { Бумага/картон } \\
\text { Paper/carton }\end{array}$ & +++ & - & - \\
\hline & $\begin{array}{l}\text { Стекло } \\
\text { Glass }\end{array}$ & +++ & - & - \\
\hline & $\begin{array}{l}\text { Резина } \\
\text { Rubber }\end{array}$ & +++ & + & ++ \\
\hline
\end{tabular}

Условные обозначения:

- Не подходит для перечисленных материалов / Not suitable for the material listed

+ Подходит для перечисленных материалов/ Suitable for the material listed

++ Очень хорошо подходит для перечисленных материалов/Well-suited for the material listed

+++ Наилучшим образом подходит для перечисленных материалов / Best suited for the material listed 
Струйная печать может осуществляться непосредственно на каких-либо частях изделия или на этикетках, которые затем прикрепляются к продукции. Автоматические этикеточные машины обычно выполняют эту операцию непосредственно во время процесса производства. Для термотрансферной печати обычно вместо чернил для создания маркировки на детали или этикетке используется термотрансферная пленка. С помощью печатающих систем можно наносить изображения, буквенно-цифровые тексты и штрих-коды на продукцию. Недостатком этих систем часто является зависимость от геометрии детали, размера маркируемой площади или сложности прикрепления этикетки.

\section{КРИТЕРИИ ВЫБОРА СИСТЕМ ЛАЗЕРНОЙ МАРКИРОВКИ ПО СРАВНЕНИЮ \\ С ДРУГИМИ МЕТОДАМИ МАРКИРОВКИ}

Выбор технологии маркировки для нанесения серийных номеров, фирменной символики или отслеживания продукции в значитель ной мере зависит от конкретной маркируемой поверхности, так как не все методы подходят для различных видов поверхностей. Ниже будут рассмотрены различные процессы лазерной маркировки, подходящие для разных видов поверхностей, а также различные методы маркировки с указанием цен и описанием их недостатков.

Технология, лежащая в основе лазерной мар кировки, является стандартной и утверждена для большинства организаций в автомобильной, аэрокосмической и оборонной промышленности. Другие системы маркировки, такие как ударно-точечная, с использованием игольчатых инструментов, термотрансферные, струйная печать и печать этикеток также основаны на надежных стандартах. Однако ударно-точечная маркировка, маркировка с использованием игольчатых инструментов и печатающие системы имеют определенные физические и технические ограничения, когда речь идет об очень мелких надписях или автоматически считываемых штрих-кодах, в то время как лазерная маркировка обеспечивает высококонтрастную маркировку с превосходным разрешением.

\section{ПРЕИМУЩЕСТВА ЛАЗЕРНОЙ МАРКИРОВКИ}

Лазерные маркировочные системы могут создавать отдельные символы, сложные изображения и штрих-коды, которые сохраняются на про- such as graphics, text, 1D and 2D codes are placed directly on the surface that needs to be marked.

Several specific marking processes are available with the use of different laser technologies and power levels, surface etching, engraving, surface color modifications, localized burning and carbonization, coating removal, etc.

\section{Dot Peen}

Marking by Dot Peen is accomplished by a mechanical movement of a hardened or carbide needle or peen. Each strike of the needle creates a dot on the surface. The placement of the dots is used to create alphanumeric text, bar codes, or other graphics. This method is only suitable for extremely robust working pieces such as steel and aluminium.

\section{Stylus Marking}

Stylus Marking of parts is similar to Dot Peen in that it uses a hardened or carbide needle to mark the surface of the part. Instead of creating individual dots, the stylus is dragged across the surface leaving a continuous mark. Alphanumeric text, bar codes, or other graphics are created by controlling where the stylus is applied to the surface. Stylus marking is used on metal, plastic, and other materials.

Printing Systems: Ink-Jet, Label, Thermo Transfer

Printing systems do not alter the surface of marked pieces. These methods place the desired data by means of a medium such as ink, label or thermal film on the working pieces. Ink-jet printing can be done directly on some parts or on a label medium which is then affixed to the part. Automated labelling machines usually perform this operation during the manufacturing process. Thermal Transfer printing rather, uses a thermal film instead of liquid ink to create the mark on the part or a label. Printing Systems can place a wide variety of graphics, alphanumeric text, and bar codes on parts. The limitation of these systems is often in the part being marked, its geometry, size of the marking area or where to affix the label.

\section{DECISION CRITERIA FOR LASER MARKING VERSUS OTHER LABELLING METHODS}

The selection of marking technology for serialization, branding, or backtracking depends significantly on the specific marked surface, as not all methods work for all surfaces. Various procedures for laser marking and the different surfaces associated with 
тяжении всего срока службы любых изделий. Лазерная маркировка обладает следующими преимуществами:

- Прямая маркировка деталей - постоянная маркировка обрабатываемой детали, прочная, абразивостойкая, устойчивая к механическим воздействиям, а также воздействию воды, растворителей, масла, температурных изменений и ультрафиолетового излучения.

- Позволяет наносить маркировку на практически любые виды поверхностей как хрупких, так и прочных изделий без физического контакта.

- Универсальность - возможность нанесения символов, текстов, кодов или изображений.

- Соответствует высочайшим гигиеническим стандартам и обеспечивает стерильность.

- Обеспечивает эффективность производства большого количества продукции с помощью автоматизированных систем.

- Исключает дополнительные издержки, расходные материалы и инструменты.

- Экологически безвредная технология: так, не используются растворители и клеящие материалы, отсутствуют отходы.

- Исключает возможность подделки и удаления.

В технологиях автомобильной промышленности стоят сложные задачи идентификации изделий на протяжении всего их срока службы, что требует маркировки на гладких или шероховатых поверхностях с очень малой площадью. Для этой области применения лазерные маркировочные устройства представляют собой оптимальное решение, так как позволяют размещать стойкую маркировку высокой плотности Datalogic
Таблица 4. Линейка продукции для лазерной маркировки компании

Table 4. Datalogic Laser Marking Product Range

\begin{tabular}{|c|c|c|}
\hline & $\begin{array}{c}\text { Универсальные устройства } \\
\text { All-in-One }\end{array}$ & $\begin{array}{c}\text { Контроллер \& головка для дис- } \\
\text { танционного сканирования } \\
\text { Controller \& remote scanning head }\end{array}$ \\
\hline $\begin{array}{l}\text { Волоконный } \\
\text { лазер } \\
\text { (1,0 мкм) } \\
\text { Fiber Laser } \\
(1.0 \mu \mathrm{m})\end{array}$ & $\begin{array}{l}\text { UNIQ } \\
\text { Первое сверхкомпактное } \\
\text { универсальное волоконное } \\
\text { лазерное маркирующее } \\
\text { устройство (15 Вт), отлича- } \\
\text { ющееся простотой исполь- } \\
\text { зования и установки, легкое } \\
\text { и очень компактное. } \\
\text { The first, ultra-соmpact } \\
15 \text { W All-in-One fiber laser } \\
\text { marking device, easy to use, } \\
\text { easy to install, light and very } \\
\text { compact. }\end{array}$ & $\begin{array}{l}\text { AREX } \\
\text { Простое решение для исполь- } \\
\text { зования в качестве автономной } \\
\text { системы или полностью автома- } \\
\text { тизированного участка в составе } \\
\text { производственной линии; } \\
\text { волоконные лазеры мощностью } \\
10 \text { Вт, } 20 \text { Вт, } 30 \text { Вт и } 50 \text { Вт. В насто- } \\
\text { ящее время имеются модели } \\
\text { волоконных лазеров с новой } \\
\text { технологией MOPA. } \\
\text { Easy solution as Stand-Alone } \\
\text { system or fully automated work } \\
\text { сеnter in production lines as } 10 \text { W, } \\
20 \text { W, } 30 \text { W and } 50 \text { W fiber laser. } \\
\text { Now available with new MOPA } \\
\text { fiber source. }\end{array}$ \\
\hline $\begin{array}{l}\mathrm{CO}_{2} \text {-лазер } \\
(10,6 \text { мкм }) \\
\mathrm{CO}_{2} \text {-Laser } \\
(10.6 \mu \mathrm{m})\end{array}$ & $\begin{array}{l}\text { ЕОХ } 10 \text { Вт } \\
\text { Универсальное решение } \\
\text { мощностью } 10 \text { Вт. Широчай- } \\
\text { шие возможности для инте-- } \\
\text { грации в производственные } \\
\text { линии, а также для исполь- } \\
\text { зования в качестве авто- } \\
\text { номной системы. } \\
\text { A } 10 \text { W All-in-One solution. } \\
\text { Offers highest flexibility } \\
\text { through integration in } \\
\text { production lines and as a } \\
\text { Stand-Alone-system. }\end{array}$ & $\begin{array}{l}\text { ЕОХ } 30 \text { Вт } \\
\text { Мощность } 30 \text { Вт, отдельная мар- } \\
\text { кирующая головка и система } \\
\text { управления; универсальность } \\
\text { использования как в качестве } \\
\text { автономной системы, таки для } \\
\text { интеграции в производственные } \\
\text { линии. } \\
\text { With } 30 \mathrm{~W} \text {, separate marking head } \\
\text { and control system, this offers } \\
\text { the highest flexibility for Stand- } \\
\text { Alone-Systems or integration into } \\
\text { production lines. }\end{array}$ \\
\hline $\begin{array}{l}\text { Твердотель- } \\
\text { ный лазер } \\
\text { (DPSS-Laser) } \\
\text { (1 064, } 532 \\
\text { и } 355 \text { нм) } \\
\text { Solid State } \\
\text { Laser (DPSS- } \\
\text { Laser) (1064, } \\
532 \text { and } \\
355 \text { nm) }\end{array}$ & $\begin{array}{l}\text { ULYXE } \\
\text { Самая компактная лазер- } \\
\text { ная маркирующая система } \\
\text { мощностью } 6 \text { Вт. UІухе-это } \\
\text { наилучшее соотношение } \\
\text { цены и производительно- } \\
\text { сти при обработке пласт- } \\
\text { массовыхи металлических } \\
\text { поверхностей. } \\
\text { The smallest } 6 \text { W laser } \\
\text { marking system. Ulyxe offers } \\
\text { the best price \& performance } \\
\text { ratio on plastics and metal. }\end{array}$ & $\begin{array}{l}\text { Серия VLASE } \\
\text { Модели с разной длиной волны- } \\
\text { 1064, } 532 \text { и } 355 \text { нм и мощностью } \\
\text { до } 20 \text { Вт. Высокая стабильность } \\
\text { при маркировке труднообраба- } \\
\text { тываемых материалов и в слож- } \\
\text { ных процессах. } \\
\text { Available in various wavelengths } \\
\text { of } 1064,532 \text {, and } 355 \text { nm for a } \\
\text { performance range up to } 20 \text { W. } \\
\text { For high stability, hard to mark } \\
\text { materials and process. }\end{array}$ \\
\hline
\end{tabular}

the relative methods will be described below, along with their costs and limitations.

The technology behind laser marking is standardized and approved by major automotive, aerospace, military and defense organizations and industries. Other marking systems, such as stylus marking, dot peen, thermal transfer, inkjet and label printing systems are also based on reliable standards. However, stylus marking, 
на поверхности деталей. Маркировка шарикоподшипников, требующая высочайшей точности, является прекрасным примером высоких требований, предъявляемых к лазерным технологиям. На протяжении всего срока службы шарикоподшипники подвергаются воздействию очень жесткой среды, включая повышенную влажность, загрязнения, повышенную температуру и различные соли. В настоящее время наблюдается массовый отзыв продукции, а прямая маркировка (DPM) гарантирует отслеживание маркированных деталей до истечения срока службы. Другие технологии маркировки, например, струйная печать, печать этикеток, термотрансферная или тампопечать не отвечают этим требованиям.

Сегодня требования, предъявляемые к отслеживанию производственного процесса на протяжении всей цепи поставок являются более строгими, чем когда-либо. Рассмотрим пример приборной доски автомобиля: каждый подсвечиваемый переключатель и инструмент маркируются лазерным устройством, в результате чего получается тонкий темный слой с прозрачным фоном. В салоне автомобиля находится printing systems and dot peening have physical and technical constraints when it comes to very small inscriptions or machine-readable bar codes, while laser marking delivers high contrast marking with fine resolution.

\section{ADVANTAGES OF LASER MARKING}

Laser marking systems create single signs, complex graphics and bar codes that are permanent over the entire life of all kinds of objects. The advantages of laser marking include:

- Direct Part Marking - permanent on the work piece, stable, abrasion-proof, resists mechanical exposure, water, solvent, oil, temperature changes and UV exposure

- Marking on almost any kind of surface without any physical contact - from fragile to massive work pieces

- Flexible use as signs, text, codes or graphics

- Adheres to highest hygienic and aseptic design requirements

- Efficient production in high quantities through automation

- No extra cost, no consumables or tools that wear out 
множество деталей, маркированных с помощью прямой маркировки с использованием лазер ных устройств. Почти каждый соединитель и розетка имеют лазерную маркировку в целях кодирования.

При производстве пищевых продуктов и напитков лазерная маркировка также является распространенным стандартом. Некоторую продукцию необходимо отдельно маркировать для обозначения срока годности. Продукция в этих отраслях требует индивидуальной маркировки на таких материалах, как бумага, метал, стекло, пластмасса или органические материалы. Преимущества лазерной маркировки совершенно очевидны. В этих областях применение технологии лазерной маркировки отличается универсальностью и высокой скоростью, может адаптироваться в соответствии с требованиями заказчика и осуществляться непосредственно на автоматической линии в процессе производства, что повышает его эффективность и обеспечивает соответствие самым строгим гигиеническим требованиям.

В производстве медицинских приборов и имплантов использование лазерной маркировки отвечает требованиям стерильности, обеспечивает защиту от подделок и размещение на деталях информации высокой плотности, причем исключается механическое воздействия и деформация материала подложки. Коды Data Matrix могут размещаться на различных поверхностях, имеющих самую разную геометрическую форму и отличаются максимальной устойчивостью к содержащим алкоголь и прочие сильнодействующие моющие средства, использующиеся в медицинских учреждениях и хирургических отделениях.

K преимуществам, которые говорят в пользу выбора лазерной маркировки в некоторых отраслях, относятся следующие:

- маркировка в автомобильной промышленности повышает эффективность производства;

- маркировка с точностью до миллиметра электронных деталей в промышленности, в частности, автомобильной;

- гибкая, программно-управляемая маркировка упаковки в пищевой промышленности и розничной торговле для обозначения срока годности и даты производства;

- высочайшая стерильность, контрастность и точность маркировки хирургических инструментов;
- Environmentally beneficial, uses no solvents or adhesives and creates no waste products

- Resistant to counterfeiting and cannot be removed

The automotive industry faces unique challenges for lifetime item identification requiring marking on smooth or rough surfaces with very little surface space. In these types of applications, laser marking devices offer an optimal solution by placing high density permanent marks directly on the component body. Precision ball bearings are an excellent example of high demands on marking technologies. Throughout their product life cycle they are exposed to harsh conditions including humidity, dirt, intense heat and salt. In times of mass product recalls, Direct Part Marking (DPM) guarantees end-of-life traceability of the marked items. Other marking technologies such as ink jet, printed label, thermal-transfer or pad printing do not stand up to these demands.

Today, requirements are more stringent than ever for tracking the production process over the entire supply chain. A car dashboard can be used as example: each backlit switch and instrument has been marked by a laser marking device with a thin dark layer exposing a light transparent background. The engine compartment of the car includes many items marked by DPM using laser marking. Almost every plug connector and socket have been laser marked for coding purposes.

In the food \& beverage Industries, labelling by laser marking is also a diffused standard. Several items require individual best-before date marking. Products need individual marking on limitless types of surfaces such as paper, metal, glass, plastic or organic material. The benefits of laser marking are clear. In these applications, laser marking is flexible, individual, applied quickly and can be performed directly on the production line automatically, improving production efficiency and complying with strict hygienic requirements.

In medical device and human implant manufacturing, the adoption of the laser marking process fulfills the requirements of aseptic design, protection against forgery and high density of information, with no mechanical interaction, deformation of modification of the substrate material. Data Matrix codes can be placed on a variety of different surfaces in several geometric shapes with maximum resistance against cleaning alcohol and other harsh cleaners typical of medical and surgical environments. 
- постоянная маркировка упаковок фармацевтических средств;

- прочная маркировка продукции на микроскопических поверхностях для борьба с контрафакцией;

- маркировка электронных компонентов в соответствии с требованиями заказчика.

Чрезвычайно важно, чтобы для каждого отдельного материала выбирался лазер с соответствующей длиной волны. Например, $\mathrm{CO}_{2}^{-}$ лазер не может наносить гравировку на металлическую поверхность, но может обрабатывать органические материалы. Подобным же образом, волоконный лазер не может маркировать органические материалы, но может обрабатывать металл. В течение долгого времени основным недостатком, препятствующим распространению лазерной маркировки, была начальная цена оборудования. В настоящее время, несмотря на то, что лазерные маркировочные устройства, как правило, являются более дорогостоящими по сравнению с принтерами для этикеток или струйными принтерами, с учетом полных затрат владельца (ТСО), включающих расходные материалы, затраты на техническое обслуживание и простои оборудования, а также утилизацию отходов, картина выглядит совершенно иначе. Это возможно благодаря повышению эффективности производства и практически нулевым затратам на расходные материалы и техническое обслуживание.

Лазерная маркировка не всегда оказывается наилучшей технологией для некоторых приложений. В случаях, когда требуется скорость и постоянство процесса, непрерывно работающие системы струйной печати все еще остаются идеальным решением, даже если лазерные маркирующие устройства и составляют им успешную конкуренцию на этом рынке.

\section{ЛАЗЕРНАЯ МАРКИРОВКА РАЗЛИЧНЫХ МАТЕРИАЛОВ (ТАБЛ. 3 И 4)}

В зависимости от обрабатываемого материала, требуются различные технологии лазерной маркировки. В табл.3 приводятся некоторые материалы и рекомендуемые лазерные системы для них. Указывается также соответствующая модель лазерного маркирующего устройства производства Datalogic. Стабильность пространственно-временных характеристик излучения лазеров дает возможность точно дозировать энергию пучка и позволяет формировать четкие
A few of the many capabilities that make laser marking the preferred choice in several industrial activities are:

- Production efficient marking in the automotive industry

- Precise marking to the millimeter of industrial or automotive electronic components

- Flexible, software-controlled laser marking for food and retail industry packaging in the case of best-before date \& manufacturing data

- Highly sterile, high-contrast, and precise marking of surgical instruments

- Continuous labelling of pharmaceutical packaging

- Microscopic size, durable marking of products to combat piracy

- Electronic component branding and personalization

It is imperative that the correct laser with the appropriate wavelength must be selected for each material. For example, a CO2-Laser cannot engrave metal, but can engrave organic material. Likewise, a fiber laser cannot mark organic material but can mark metal.

For a long time, the main obstacles preventing the diffusion of laser marking was the initial cost of the apparatus. Now, despite the fact that laser marking devices are typically more expensive than label printers or than continuous inkjet printers, the TCO, (Total Cost of Ownership, that includes consumables, maintenance cost and downtime, waste disposal etc.) may offer a completely different outlook. This is possible due to increased production efficiency with almost zero consumption and maintenance costs.

Laser marking is not always the best technology choice for every application. At extremely high speeds and marking-on-the-fly applications, continuous ink-jet systems are still ideal, even if some manufacturers of specialty laser marking devices have become very competitive in this market.

\section{LASER MARKING FOR VARIOUS MATERIALS}

Depending on the material to be processed, various laser technologies are required for marking. The chart below lists several materials and the recommended laser system to be used for each. The corresponding Datalogic laser marking model is shown.

Laser Marking Systems - the right solution for every requirement 
структуры на поверхности с небольшим разбросом геометрических параметров.

Системы лазерной маркировки - лучшее решение, отвечающее всем необходимым требованиям.

Постоянно наблюдаемый прогресс в области программного обеспечения лазерных маркеров обеспечивает конкурентоспособность лазерных методов маркировки по сравнению с традиционными методами маркировки.

Компания Datalogic предлагает различные лазерные технологии для конкретных областей применения для использования в качестве автономных систем или интеграции в производственные линии. В настоящее время во всем мире используются около двадцати тысяч лазерных маркировочных систем Datalogic (табл. 4).

Наряду с аппаратным оборудованием при принятии решения в отношении выбора лучшей технологии следует учитывать также и программное обеспечение. Datalogic предлагает уникальный комплект программного обеспечения для лазерной маркировки, который специально разработан для обеспечения бесперебойной работы со всеми устройствами лазерной маркировки компании Datalogic независимо от используемой лазерной технологии или конфигурации системы.

Lighter Software Suite обеспечивает усовершенствованное редактирование изображений и полный контроль над всеми параметрами лазера, включая управление координатной осью, программируемый цифровой ввод-вывод и диагностику. Мощный механизм выполнения сценария позволяет адаптировать программный интерфейс к специфическим потребностям пользователя или к системной среде. Lighter Software Suite позволяет непосредственно проектировать и осуществлять взаимодействие с обычными промышленными базами данных или системами предприятий, например SAP, или со сканерами штрих-кодов, мобильными компьютерами или системами обработки изображений.

Брэнд Datalogic и его логотип являются зарегистрированными торговыми знаками компании Datalogic S.p.A. во многих странах, включая США и Европейский Союз. Лozomunb Datalogic ADC u Datalogic Automatic Data Capture - торговыми марками компании Datalogic S.p.A. Больще информащии можно получить на сайте www.datalogic.com.
Datalogic offers various laser technology solutions for individual applications as stand-alone systems or as integrations into production lines. About twenty thousand laser marking systems from Datalogic are currently in use worldwide.

Along with the hardware, laser marking software must be kept in mind when making a decision on the best solution to adopt. Datalogic offers a unique marking software suite, developed to work seamlessly with all Datalogic marking products regardless of the laser technology or system configuration used.

Lighter Software Suite allows advanced graphics editing and full control of all laser parameters, included axis control, programmable digital I/O and diagnostics. A powerful built-in script engine allows the software interface to be adapted to the user's specific needs or to the system environment. Interaction with common production databases or enterprise systems, such as SAP, or with bar code readers, mobile computers or image processing systems can be directly designed and implemented in a Lighter Software Suite environment.

Datalogic Group is a global leader in Automatic Data Capture and Industrial Automation markets. As a world-class producer of bar code readers, mobile computers, sensors for detection, measurement and safety, vision systems and laser marking systems, Datalogic offers innovative solutions for a full range of applications in the retail, transportation \& logistics, manufacturing and healthcare industries. With products used in over a third of world's supermarkets and points of sale, airports, shipping and postal services, Datalogic is in a unique position to deliver solutions that can make life easier and more efficient for people. Datalogic S.p.A., listed on the STAR segment of the Italian Stock Exchange since 2001 as DAL.MI, is headquartered in Lippo di Calderara di Reno (Bologna). Datalogic Group as of today employs about 2,500 members of staff worldwide distributed in 30 countries. In 2015 Datalogic Group achieved revenues for 535,1 million Euro and invested over 48 million Euro in Research and Development with a portfolio of about 1,200 patents and pending patent applications in multiple jurisdictions. For more news and information on Datalogic, please visit www.datalogic.com.

Datalogic and the Datalogic logo are registered trademarks of Datalogic S.p.A. in many countries, including the U.S.A. and the E.U. Datalogic ADC is a trademark of Datalogic S.p.A. All other brand and product names may be trademarks of their respective owners. 